\title{
Ductus Arteriosus Doppler in der ersten Schwangerschaftshälfte
}

Ch. Brezinka, J.W. Wladimiroff, T. Huisman, Th. Stijnen ${ }^{2}$

Frauenklinik der Erasmusuniversität Rotterdam, Acad. Ziekenhuis Dijkzigt, Dr. Molewaterplein 40, N-3015 GD Rotterdam

2Institut für Biostatistik der Erasmusuniversität Rotterdam

\section{Einleitung, Material und Methoden}

Der fetale ductus arteriosus (DA) ist in den letzten Jahren für Geburtshelfer zunehmend interessant geworden (1). Wir haben versucht, den DA vom frühest möglichen Zeitpunkt der Schwangerschaft an zu messen und eine Normkurve für die Flow-Parameter zu erstellen. Reproduzierbare Messungen mit der Vaginalsonde gelangen ab der 11. SSW. Insgesamt wurden 256 Frauen zwischen der 9. und 25. SSW in die Untersuchnung aufgenommen, nach Abzug aller mißlungenen und undeutlichen DopplerKurven blieben 174 zur weiteren Analyse. Gemessen wurde in der hohen parasternalen kurzen Achse, damit lag der Schallkopf in der Ebene der Blutflußrichtung. Folgende Flow-Parameter wurden bestimmt: Die systolische Spitzengeschwindigkeit, die mittlere Geschwindigkeit, die enddiastolische Geschwindigkeit. Aus diesen Parametern wurde der Pulsatilitätsindex (PI) errechnet. Weiter wurden das Flußintegral, die Dauer einer Herzaktion (Period Time) sowie die Beschleunigungszeit, von Beginn bis zum Höhepunkt des Systole bestimmt.

\section{Ergebnisse}

Die maximalen systolischen Flußgeschwindigkeiten betragen in der 11. SSW ca. 30 $\mathrm{cm} / \mathrm{sec}$. Dieser, Wert steigert sich bis zur $20 \mathrm{SSW}$ kontinuierlich auf $80 \mathrm{~cm} / \mathrm{sec}$ an (Abb. 1). Damit zeigt sich, daß der DA während der gesamten Schwangerschaft das „schnellste“ Blutgefäß ist (2). Auch bei den mittleren Flußgeschwindigkeiten wurde eine kontinuierliche Steigerung festgestellt.

Enddiastolischer Flow ist ab der 13. SSW sporadisch und ab der 16 SSW immer meßbar. Dies entspricht Messungen in der A. Umbilicalis und der Aorta, wo ebenfalls

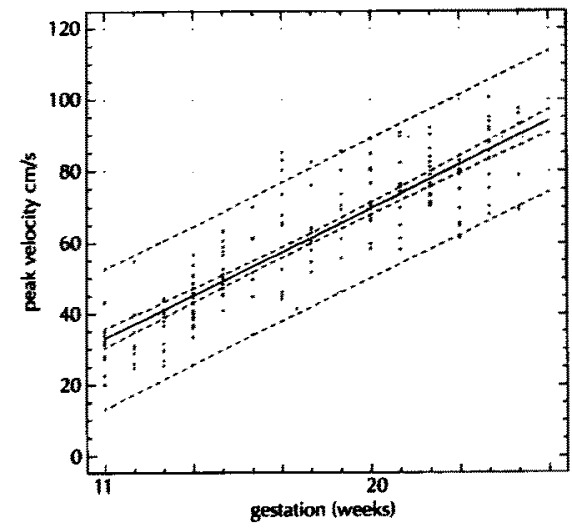

Abb. 1. Die maximalen systolischen Flußgeschwindigkeiten des Ductus Arteriosus von der 11 bis zur 24 SSW dargestellt mit STATGRAPHICS 
enddiastolischer Blutfluß ab der 13/14. SSW gemessen wird (3). Das Integral, d.h. die Fläche unter der Dopplerkurve, steigt ebenfalls kontinuierlich an. Wenn wir in der von uns beobachteten Gestationszeit eine stets maximale Dilatation des Ductus annehmen, so kann aus den Werten auf einen kontinuierlichen Anstieg des rechtsventrikulären Auswurfvolumens geschlossen werden. Die Period Time nimmt kontinuierlich zu, ebenso wird die Beschleunigungszeit länger. Der Pulsatilitätsindex bleibt im Beobachtungszeitraum gleich, zwischen 2 und 3 und einem Mittel bei 2,6. Unter Indomethacinbehandlung wird PI unter 1,9 als Hinweis auf Ductus-Konstriktion angesehen (4).

\title{
Zusammenfassung
}

Zusammenfassend stellen wir fest, daß es möglich ist, Flußgeschwindigkeiten durch den ductus arteriosus ab der 11. SSW zu messen. Enddiastolischer Flow tritt an der 13. SSW auf, vermutlich aufgrund abnehmender Afterload. Sowohl eine Abnahme der peripheren Afterload (Plazenta) als auch eine Zunahme der rechtsventrikulären Kontraktilität und das Auswurfvolumen sind für den Anstieg aller Blutflußgeschwindigkeiten im ductus arteriosus verantwortlich.

\section{Literatur}

1. Tulzer,G., Gudmundsson,S., Sharkey,A.M., Wood,D.C., Cohen,A.W., Hutha,J.C.: Doppler echocardiography of fetal ductus arteriosus constriction versus increased right venticular output J. Am. Coll. Cardiol. 18, 532-536, 1991

2. Mooren van der,K., Barenregt,L.G., Wladimiroff,J.W.: Flow velocity wave forms in the human fetal ductus arteriosus during the normal second half of pregnancy Pediatr. Res. 30, 487490, 1991

3. Wladimiroff,J.W., Huisman,T.W:, Stewart,P.A.: Fetal and umbilical flow velocity waveforms between 10-16 weeks gestation: a preliminary study Obstet Gynecol. 78, 812-814, 1991

4. Tulzer,G., Gudmunsson, Tews,G., Wood,D.C., Huhta,J.C.: Incidence of indomethacin-induced human fetal ductal constrictions J. Matern Fetal Invest 1, 267-269, 1992

\section{Stellenwert der Biometrie des fetalen Herzens im II und II Trimenon der Schwangerschaft}

\author{
K.S. Heling, R. Chaoui, R. Bollmann
}

Abteilung Pränatale Diagnostik und Therapie, UFK Humboldt - Universität (Charité) Berlin, Schumannstr. 20/21, D-10117 Berlin

\section{Einleitung}

Angebore Herzfehler stellen mit einer Inzidenz von $0,7-0,9 \%$ die häufigsten kongenitalen Fehlbildungen dar. Im Rahmen der pränatalen Diagnostik des fetalen Herzens dient die Ermittlung der Biometrie mittels charakteristischer Parameter vor allem zur Beurteilung des normalen Wachstums und der Erkennung von Normabweichungen. Das Ziel der retrospektiven Studie bestand in der Erstellung eigener Normkurven für verschiedene biometrische Parameter des fetalen Herzens. 